\title{
NOUVELLE
}

\section{Une nouvelle population de cellules dendritiques sanguines aux propriétés particulières vis-à-vis du VIH-1}

Flavien Brouiller ${ }^{1,2}$, Nicolas Ruffin ${ }^{1}$, Philippe Benaroch ${ }^{1}$
> En 2018, environ 38 millions de personnes dans le monde vivaient infectées par le virus de l'immunodéficience humaine (VIH), dont 1,7 million de personnes nouvellement infectées (source ONUSIDA). En dépit des politiques de prévention et de l'efficacité des nouveaux traitements anti-rétroviraux, l'infection par le VIH demeure un enjeu majeur de santé publique.

Le VIH infecte des populations de cellules qui expriment à leur surface la molécule CD4, nécessaire à l'entrée du virus : lymphocytes $\mathrm{TCD}^{+}$, macrophages, et cellules dendritiques (dendritic cells, DC). Ces dernières ont un rôle clé dans la réponse anti-virale. En effet, elles ont la capacité de rapidement détecter la présence de microorganismes pathogènes, et de produire alors des cytokines. Elles sont également cruciales pour la mise en place de la réponse immunitaire adaptative, en présentant des antigènes dérivés des pathogènes aux lymphocytes $T$, ce qui déclenche leur activation [1]. Les DC sont moins susceptibles à l'infection par le VIH que les lymphocytes $\mathrm{T} \mathrm{CD}^{+}$ou les macrophages, mais seraient néanmoins aussi impliquées dans la propagation de l'infection par transmission du VIH à d'autres cellules [2, 3]. II est désormais admis que la transmission du virus de cellule à cellule est très efficace [4]. La transmission du virus par les DC peut s'effectuer, soit directement par contact entre cellules et passage de virions d'une DC infectée à une cellule voisine (infection « en cis »), soit après capture, sans infection, des particules virales par la DC, qui les transmet ensuite à d'autres cellules par contact (infection « en trans ») [2]. Dans ce cas, les DC capturent les particules virales grâce à leur récepteur Siglec-1 (CD169), qui présente une forte affinité pour des gangliosides abondants sur l'enveloppe des virions [5].
${ }^{I}$ Institut Curie, PSL* research university, Inserm U932, 12 rue Lhomond, 75005 Paris, France.

${ }^{2}$ Sorbonne université, عD394, Paris, France. philippe.benaroch@curie.fr

L'hétérogénéité des $D C$ présentes dans le sang a pu être finement analysée récemment grâce aux techniques de protéomique et de transcriptomique sur cellule unique. Ces études ont révélé la présence d'une nouvelle population de $D C$ exprimant Siglec-1 de façon constitutive, appelées pré-DC (ou AS-DC) [6, 7], qui seraient des précurseurs des $D C$ conventionnelles ( $C D C$ ), également présentes dans le sang. Ces dernières contiennent deux sous-populations de cellules ( $c D C 1$ et $c D C 2$ ) spécialisées dans la présentation antigénique aux lymphocytes T [1]. Enfin, les DC plasmacytoïdes ( $p D C)$ constituent une sous-population de DC spécialisées dans la production de grandes quantités d'interférons de type I en réponse à la détection de pathogènes [8]. Jusqu'ici, aucune fonction particulière n'avait été attribuée aux pré-DC. La présence de Siglec-1 à leur surface et la capacité de 

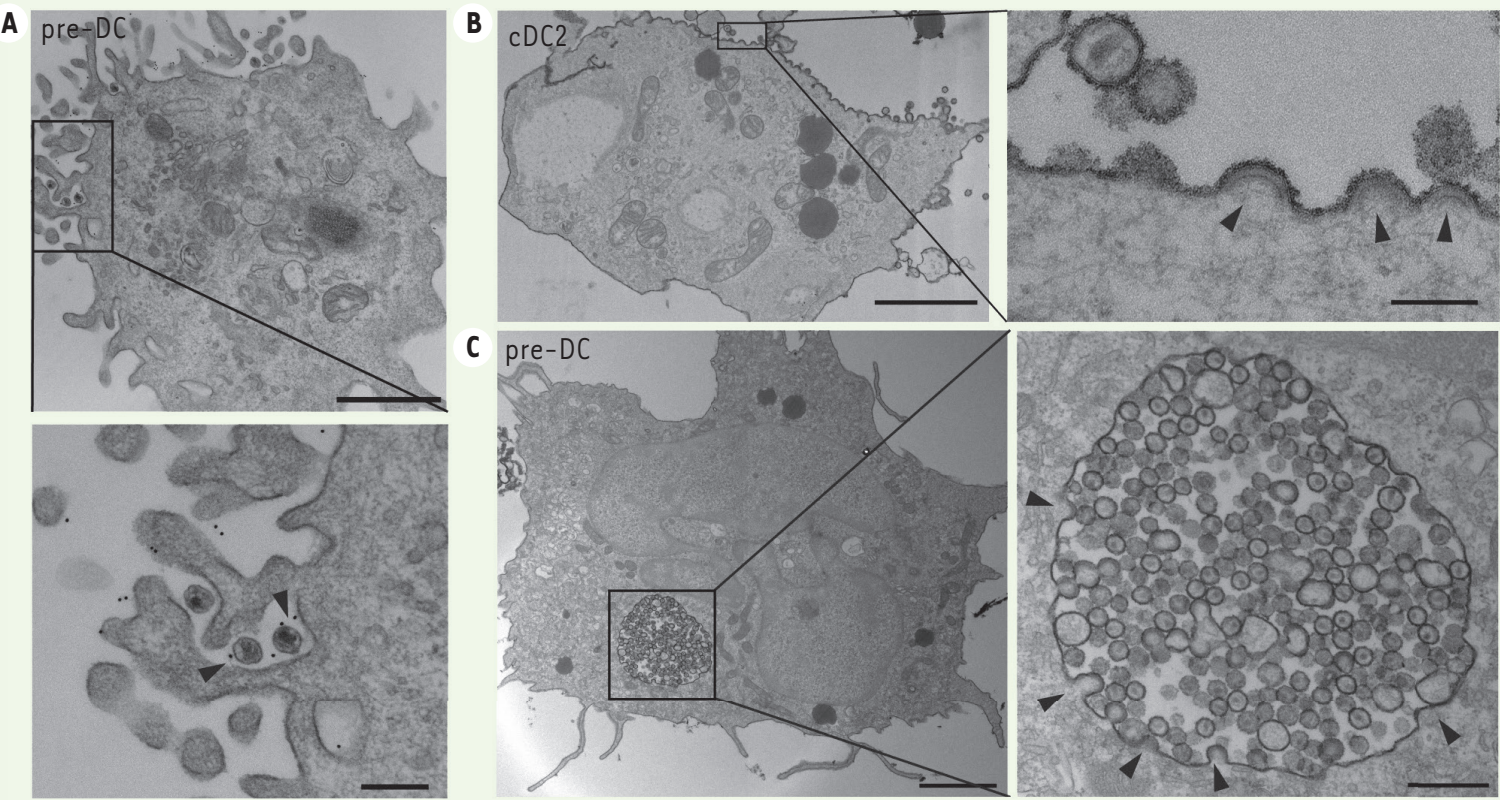

Figure 1. Les pré-DC capturent le VIH via Siglec-1 et produisent de nouveaux virions dans des compartiments d'apparence intracellulaire. A. Analyse par microscopie électronique et immuno-marquage des pré-DC exposées au VIH-l pendant deux heures, fixées, et marquées avec un anticorps monoclonal dirigé contre Siglec-1 (révélation par la protéine A couplée à des particules d’or de $5 \mathrm{~nm}$ de diamètre). Une coupe Epon (inclusion dans la résine) est représentée, avec un agrandissement en dessous. Les flèches indiquent un marquage spécifique de Siglec-l à l'interface entre le virus et les invaginations de la membrane plasmique. Barre d'échelle : $2 \mu \mathrm{m}$ (image du haut) ou 0,5 $\mu \mathrm{m}$ (image agrandie). B-C. Coupes Epon des CDC2 (B) et des pré-DC (C) infectées par le VIH-1 à 48 heures. Les cellules ont été fixées en présence de rouge de ruthénium pour marquer les membranes en contact avec le milieu extracellulaire. Les flèches indiquent des bourgeonnements viraux. Barre d'échelle : $2 \mu \mathrm{m}$ ou $0,15 \mu \mathrm{m}$ (images agrandies) (figure adaptée d'après [9]).

ce récepteur à se lier au VIH nous ont incités à analyser les relations des préDC avec le VIH et à les comparer à celles des autres sous-populations de DC [9].

\section{Les pré-DC sont susceptibles à l'infection par le VIH}

Pour cette étude, nous avons purifié, à partir d'échantillons de sang de donneurs sains, les quatre sous-populations de $D C$ définies précédemment, à savoir les pré- $D C, C D C 1, c D C 2$, et $p D C$, par une combinaison de tris par billes magnétiques et cytométrie en flux. En exposant ces sous-populations au VIH-l in vitro, nous avons montré que les pré-DC, et dans une moindre mesure les $C D C 2$, sont susceptibles à l'infection par le VIH qui utilise le corécepteur CCR5 ( $C-C$ motif chemokine receptor 5 ) pour son entrée dans ces cellules (tropisme R5). Néanmoins, seules les pré-DC sont infectées par le VIH, qui utilise alors le corécepteur CXCR4 ( $C-X-C$ motif chemokine receptor 4$)$ pour son entrée dans ces cellules (tropisme X4). Dans ce dernier cas surtout, l'infection des pré-DC est dépendante de Siglec-1, ce qui n'est pas le cas pour les $C D C 2$. Les pré-DC comme les CDC2 expriment le facteur SAMHD1 (SAM domain and HD domain-containing protein 1), qui restreint l'infection. En contrecarrant l'effet de SAMHDl grâce à la protéine Vpx du VIH-2, on augmente fortement les taux d'infection des pré-DC avec les deux types de VIH-1 (tropismes R5 et X4).

Nous montrons notamment par microscopie électronique et immuno-marquages (Figure IA) que Siglec-l est également essentiel pour la capture des particules virales par les pré-DC. En effet, l'emploi d'un anticorps spécifique de Siglec-l inhibe à la fois la capture des particules virales par ces cellules et leur infection. Une fois infectées, les pré-DC et les cDC2 sont toutes deux capables de produire des nouveaux virions, qu'elles libèrent dans le milieu extérieur.

Les sites de production virale ont été étudiés par une analyse ultra-structurale, qui a révélé qu'ils sont totalement distincts dans les deux sous-types de DC (Figures 1B, 1C). Dans les CDC2 infectées par le VIH, les bourgeonnements viraux s'effectuent de façon très polarisée et uniquement à la membrane plasmique (Figure 1B), comme c'est le cas dans les lymphocytes T CD4 ${ }^{+}$ infectés. En revanche, dans les pré-DC infectées, les sites de bourgeonnements viraux sont concentrés dans la membrane limitante de compartiments apparemment intracellulaires, contenant dans leur lumière de nombreuses 


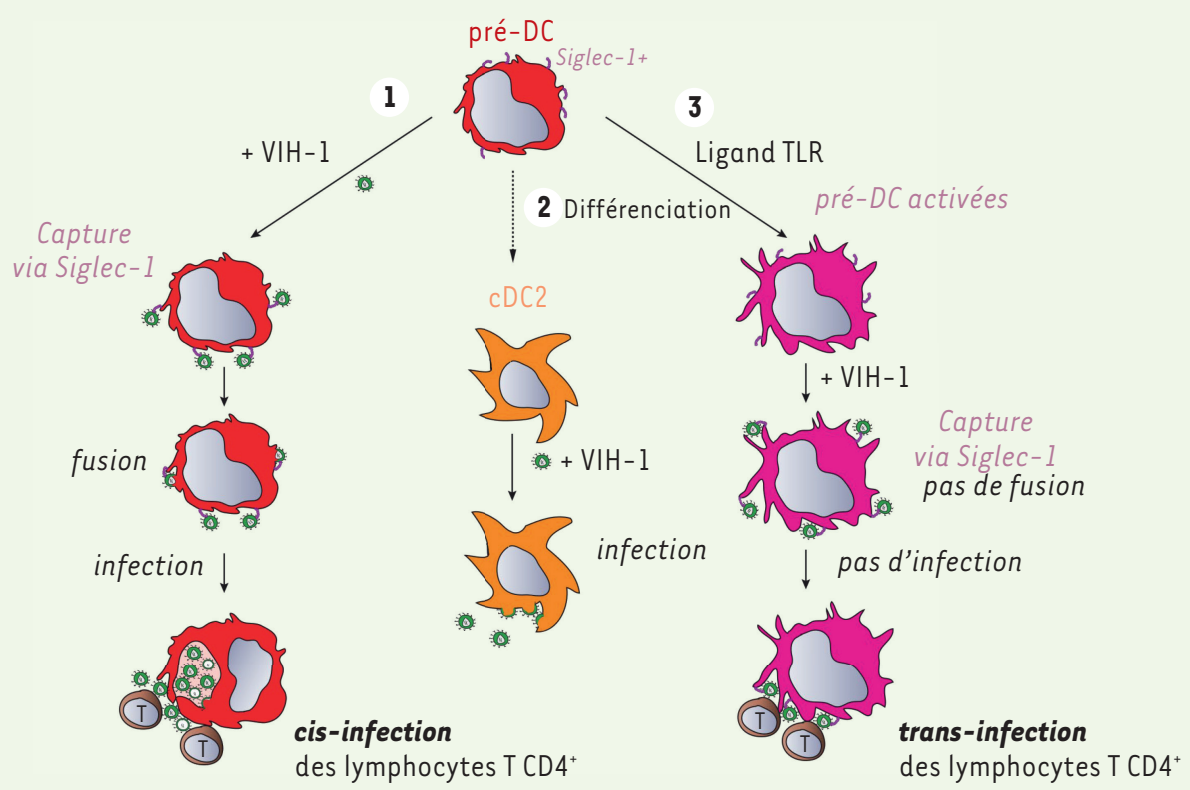

Figure 2. Les pré-DC possèdent des propriétés uniques vis-à-vis de l'infection par le VIH. Différentes situations sont observées. 1. Les préDC peuvent capturer VIH-1 via Siglec-1, fusionner avec le virus, et devenir infectées. Elles produisent alors de nouveaux virus dans des compartiments d'apparence intracellulaire. Les nouveaux virus sont efficacement transmis en cis aux lymphocytes T CD4 $4^{+}$. 2. Les pré-DC peuvent se différencier en CDC2, susceptibles à l'infection par le VIH-1 (tropisme R5). Les CDC2 infectées produisent de nouveaux virus à leur membrane plasmique. 3. Après activation, les pré-DC résistent à l'infection par le VIH-1. Elles peuvent néanmoins encore capturer les particules virales via Siglec-1, et les transmettent efficacement aux lymphocytes T CD4+ activés. TLR : Toll-like receptor (figure adaptée d'après [9]).

particules virales. Ces compartiments sont connectés au milieu extérieur, comme c'est le cas dans les macrophages infectés [10]. Cela est attesté par la présence au niveau des membranes de ces compartiments et des particules virales, d'un colorant ajouté au moment de la fixation des cellules, le rouge de ruthénium, qui souligne les membranes d'une ombre grisée, sans les traverser (Figure 1C).

Ainsi, les pré-DC exposées à des particules de VIH-l sont capables de les capturer via Siglec-1, d'être infectées, et de produire de nouvelles particules virales qui s'accumulent dans la lumière de compartiments intracellulaires de façon similaire à ce qui est observé dans les macrophages primaires infectés par le virus. Les pré-DC peuvent se différencier en cDC2 qui, après leur infection par le virus, se comportent comme des lymphocytes $T$ en produisant de nouveaux virions.
Activées ou non, les pré-DC sont capables de transmettre le VIH-1 à d'autres cellules

En raison de leur susceptibilité au VIH-l et de leur expression constitutive de Siglec-1, les pré-DC pourraient participer à la propagation du virus aux lymphocytes T CD4 ${ }^{+}$. Nous avons montré que l'infection est transmise à des lymphocytes $\mathrm{T} \mathrm{CD4}^{+}$activés lorsqu'ils sont co-cultivés avec des pré-DC infectées (infection en cis). Les pré-DC infectées sont donc capables de produire des particules virales infectieuses.

Le VIH-l parvient à fusionner avec $80 \%$ des pré-DC fraîchement purifiées. Néanmoins, il est totalement incapable de fusionner lorsque les pré-DC ont été activées au préalable par des ligands des récepteurs Toll-like. Ainsi, les pré-DC activées sont totalement résistantes à l'infection, mais elles s'avèrent toujours capables de capturer, via Siglec-1, des particules virales, qu'elles parviennent à transmettre à des lymphocytes T CD4 ${ }^{+}$ activés (infection en trans).

En employant le VIH-l comme microorganisme pathogène modèle, notre étude a permis la première caractérisation fonctionnelle d'une population de DC, les pré$D C$, jusque-là définie essentiellement d'un point de vue ontogénique. L'expression constitutive de Siglec-l par les pré-DC leur confère des propriétés uniques de capture, d'infection et de transmission du VIH-1 par rapport aux autres sous-populations de DC sanguines (Figure 2). II reste désormais à analyser le rôle des pré-DC dans la mise en place de la réponse immunitaire antivirale et dans le maintien des différentes sous-populations de DC au cours de l'infection, ainsi qu'à étudier les mécanismes de fusion avec des particules virales mis en jeu dans ces différentes sous-populations. $\diamond$

A new population of blood precursors of dendritic cells endowed with specific properties regarding HIV-1 


\section{REMERCIEMENTS}

Ces travaux de recherche ont été soutenus par les subventions suivantes, attribuées à Philippe Benaroch: ANRS (agence nationale de recherche contre le SIDA et les hépatites virales) ECTZ72276, Sidaction (Ensemble contre le SIDA) 2018-1-AEO11984, ANR (agence nationale pour la recherche) ANR-10-IDEX-0001-02 PSL et ANR-11-LABX-0043. Nicolas Ruffin a bénéficié de bourses post-doctorales de l'ANRS et de la FRM (fondation pour la recherche médicale). Flavien Brouiller bénéficie d'une bourse doctorale de Sorbonne Université.

\section{LIENS D'INTÉRÊT}

Les auteurs déclarent n'avoir aucun lien d'intérêt concernant les données publiées dans cet article.

\section{RÉFÉRENCES}

1. Schlitzer A, Mcgovern N, Ginhoux F. Dendritic cells and monocyte-derived cells: Two complementary and integrated functional systems. Semin Cell Dev Biol $2015 ; 41: 9-22$
2. Granelli-Piperno A, Delgado $\varepsilon$, Finkel V, et al. Immature dendritic cells selectively replicate macrophagetropic (M-tropic) human immunodeficiency virus type 1 , while mature cells efficiently transmit both $\mathrm{M}$ - and T-tropic virus to $\mathrm{T}$ cells. J Virol $1998 ; 72: 2733-7$.

3. Mcilroy D, Autran B, Cheynier R, et al. Infection frequency of dendritic cells and $C D 4^{+} T$ lymphocytes in spleens of human immunodeficiency virus-positive patients. J Virol $1995 ; 69: 4737-45$.

4. Sattentau Q. Avoiding the void: cell-to-cell spread of human viruses. Nat Rev Microbiol 2008 ; 6 : 815-26.

5. Izquierdo-Useros N, Lorizate M, Mclaren PJ, et al. HIV- 1 capture and transmission by dendritic cells: The role of viral glycolipids and the cellular receptor Siglec-1. PLoS Pathog 2014 ; 10 : el004146.

6. See P, Dutertre CA, Chen J, et al. Mapping the human $D C$ lineage through the integration of highdimensional techniques. Science 2017 ; 56. pii: eaag3009. doi: 10.1126/science.aag3009.

7. Villani AC, Satija R, Reynolds G, et al. Single-cell RNA seq reveals new types of human blood dendritic cells, monocytes, and progenitors. Science 2017 ; 3. pii: eaah4573. doi: 10.1126/science.aah4573.

8. Müller-Trutwin M, Hosmalin A. Role for plasmacytoid dendritic cells in anti-HIV innate immunity. Immunol Cell Biol $2005 ; 83: 578-85$.
9. Ruffin N, Gea-Mallorquí $\varepsilon$, Brouiller F, et al. Constitutive Siglec- 1 expression confers susceptibility to HIV-1 infection of human dendritic cell precursors. Proc Natl Acad Sci USA 2019; 116 : 21685-93.

10. Hammonds JE, Beeman N, Ding L, et al. Siglec-1 initiates formation of the virus-containing compartment and enhances macrophage-to- $\mathrm{T}$ cell transmission of HIV-1. PLoS Pathog $2017 ; 13$ : el006181.
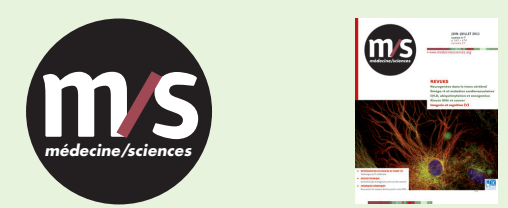

Abonnez-vous à médecine/sciences

Bulletin d'abonnement page 426 dans ce numéro de $\mathrm{m} / \mathrm{s}$ 